\title{
Temperature effect of metastable atom desorption from solid Ne by low-energy electron impact
}

\author{
Haruaki Kato ${ }^{1}$, Takayuki Tachibana ${ }^{2^{*}}$, and Takato Hirayama ${ }^{1}$ \\ ${ }^{1}$ Department of Physics, Rikkyo University, Nishi-Ikebukuro, Toshima-ku, Tokyo 171-8501, Japan \\ E-mail: hirayama@rikkyo.ac.jp \\ ${ }^{2}$ Department of Physics, Tokyo University of Science, Shinjuku-ku, Tokyo 162-8601, Japan
}

Received May 16, 2012

\begin{abstract}
We study the desorption of metastable atoms induced by electronic transitions in solid Ne by low energy electron impact. Time-of-flight spectra and the angular distribution of metastable atoms desorbed from the surface of an annealed sample show increased kinetic energy, higher signal intensity, and a narrower angular distribution compared with those measured using an unannealed sample. These results are explained by considering the sample's surface conditions in the framework of the cavity ejection mechanism. Our results for annealed solid $\mathrm{Ne}$ show that when the sample temperature increases from 5 to $7 \mathrm{~K}$, the width of the angular distribution increases by about $10 \%$. A simple trajectory calculation qualitatively reproduces our experimental results.
\end{abstract}

PACS: 79.20.La Photon- and electron-stimulated desorption;

71.35.Gg Exciton-mediated interactions.

Keywords: metastable atom, desorption, electronic transition, time-of-flight spectra.

\section{Introduction}

Desorption induced by electronic transitions (DIET) in rare gas solids by elementary excitation has been investigated over the past 20 years [1]. Cavity ejection (CE) is one of the mechanisms that leads to excited atom desorption such that an excited atom created on the surface of the solid by incident electrons or photons is repelled by surrounding atoms in the ground state and desorbs from the surface. When this mechanism is applicable, desorbed excited atoms are expected to show a narrow angular distribution that is concentrated near the surface normal direction. In real samples, this narrow angular distribution may become broader because of either imperfections in the crystal or lattice vibration, or both.

Sakurai et al. [2] measured the angular distribution of metastable atoms desorbed from the surface of solid Ne by low energy photon irradiation. They succeeded in qualitatively reproducing their experimental results using a simple trajectory calculation.

To observe the effects caused by conditions of the sample surface as well as those owing to lattice vibration, we measured time-of-flight spectra and the angular distribution of atoms desorbed from the surfaces of unannealed and annealed samples at various temperatures. We will discuss the results within the framework of the CE mechanism, and we also performed a simple trajectory calculation of desorbed excited $\mathrm{Ne}$ atoms.

\section{Experimental setup}

Figure 1 shows a side view of the experimental setup, which was similar to the one we used in our previous work [3-5], except for the addition of a low-energy electron gun and a channel electron multiplier (CEM) for the detection of desorbed metastable atoms. The main chamber was evacuated by a series of turbo molecular pumps and a Ti-getter pump, resulting in a pressure of approximately $3 \cdot 10^{-8} \mathrm{~Pa}$.

The sample film was prepared on a polycrystalline $\mathrm{Cu}$ disk of $10 \mathrm{~mm}$ diameter, which was fixed to a mechanical cryostat and cooled down to $5.0 \mathrm{~K}$. The cryostat was surrounded by a heat shield that was kept at about $40 \mathrm{~K}$. The cryostat can be rotated without breaking the vacuum, which enables us to measure the angular distribution of desorbed particles. The sample film was condensed on the $\mathrm{Cu}$ disk by filling the chamber with gaseous $\mathrm{Ne}$ to a pressure of $10^{-6}-10^{-4} \mathrm{~Pa}$. The film thickness was estimated from the exposure assuming a condensation coefficient to be unity.

* Present address: Department of Physics, Rikkyo University, Nishi-Ikebukuro, Toshima-ku, Tokyo 171-8501, Japan 


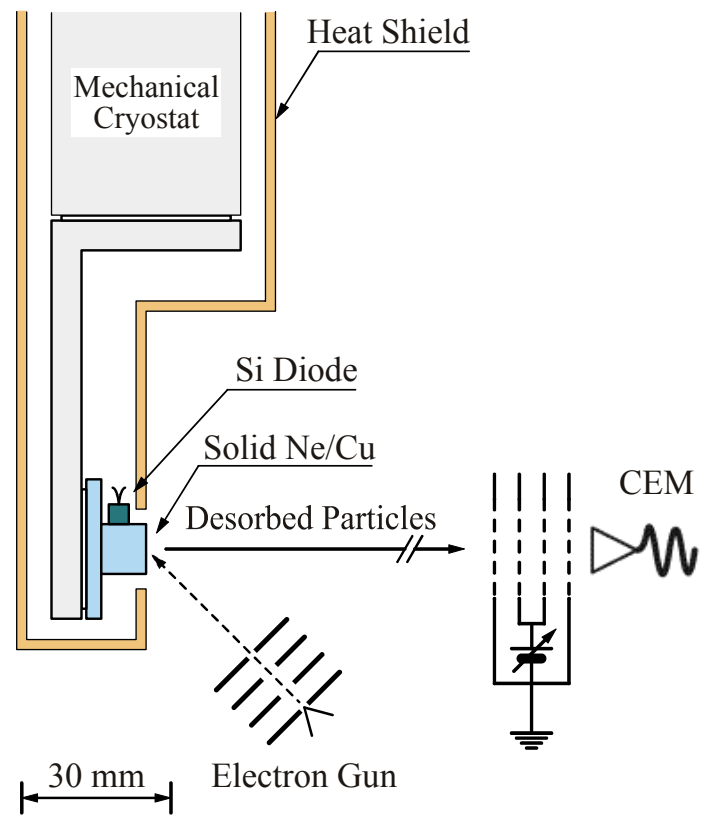

Fig. 1. (Color online) Schematic of the experimental setup (side view).

The temperature of the sample was controlled in the range between 5 and $100 \mathrm{~K}$ by a heater mounted in the cryostat. The temperature stability during the measurements was $\pm 0.1 \mathrm{~K}$. The sample temperature was measured using a Si-diode (LakeShore, DT-670A-SD) attached to the $\mathrm{Cu}$ substrate. Solid $\mathrm{Ne}$ was formed at a substrate temperature of $5.0 \mathrm{~K}$, and was annealed at $7.0 \mathrm{~K}$.

An electron gun, which produces an electron beam with an energy between 17 and $100 \mathrm{eV}$, was fixed to the heat shield, so that the incident angle of the electron beam to the sample surface was fixed during the measurements of the angular distribution. For our measurements of the timeof-flight spectra, the electron beam was pulsed by applying a pulsed voltage to the first grid of the electron gun. The width and frequency of the pulsed beam were approximately $10 \mu$ s and $500 \mathrm{~Hz}$, respectively.

Desorbed metastable atoms were directly detected by a CEM that was fixed to the chamber wall. The distance between the sample and the CEM was $287 \mathrm{~mm}$. The lifetimes of the Ne metastable states $\left(2 p^{5} 3 s^{3} P_{0.2}\right)$ are known to be much longer than the flight time in the present experiments (typically less than $1 \mathrm{~ms}$ ). Desorbed ions and emitted electrons were rejected by applying a positive voltage to the grid mesh in front of the CEM and a negative voltage to the entrance funnel of CEM, respectively.

\section{Experimental results}

\subsection{Time-of-flight spectra}

Figure 2 shows the time-of-flight spectra of metastable atoms desorbed from the surface of solid Ne measured at the sample normal direction $\left(\theta=0^{\circ}\right)$. The incident electron

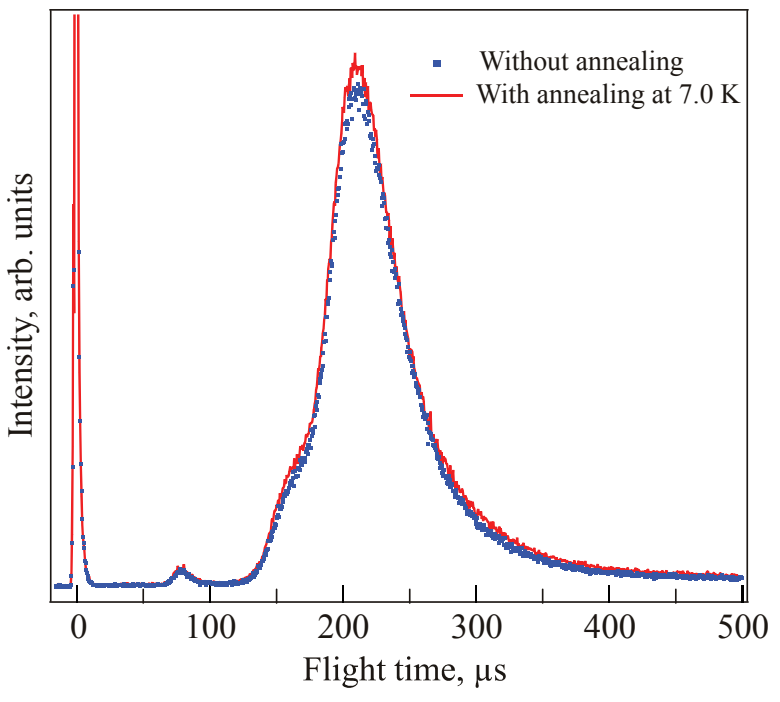

Fig. 2. (Color online) Time-of-flight spectra of metastable atoms desorbed from the surface of solid $\mathrm{Ne}$ measured in the sample normal direction $\left(\theta=0^{\circ}\right)$. The incident electron energy was $30 \mathrm{eV}$ and the thickness of the sample was 300 atomic layers. The solid line and dots represent the spectra from annealed and unannealed $\mathrm{Ne}$ solid, respectively.

energy was $30 \mathrm{eV}$ and the sample thickness was 300 atomic layers. The strongest peak at flight time $t_{f}=0$ is caused by the emitted VUV light from the sample. The higher kinetic energy peak $\left(t_{f} \sim 80 \mu \mathrm{s}, E_{k}=1.4 \pm 0.1 \mathrm{eV}\right)$ is owing to metastable $\mathrm{Ne}$ atoms that are desorbed through the excimer dissociation (ED) process. The peak at $t_{f} \sim 210 \mu \mathrm{s}$ $\left(E_{k}=0.18 \pm 0.02 \mathrm{eV}\right)$ is caused the cavity ejection (CE) mechanism.

Figure 2 shows that the CE peak of the annealed sample (solid line) exhibits a higher intensity. A slight increase of the kinetic energy $(\sim 0.6 \%)$ was also observed in the spectrum of the annealed sample. The kinetic energy did not change in the spectra of the annealed sample measured at temperatures between 5.0 and $7.0 \mathrm{~K}$ within the experimental statistical uncertainties.

\subsection{Angular distribution}

The time-of-flight spectra measured at $\theta=0,10$, and $20^{\circ}$ from the sample normal direction are shown in Fig. 3. The intensity of the CE peak significantly decreases as the angle increases. The CE peak area as a function of observation angle (angular distribution) is plotted in Fig. 4. This clearly shows that the sample that annealed at $7.0 \mathrm{~K}$ (open circles) exhibits a narrower angular distribution than the unannealed sample (solid circles).

The angular distribution of the CE peak area of the annealed sample was measured as a function of temperature. The temperature dependence of the full width at half maximum (FWHM) of the distribution is shown in Fig. 5. The FWHM increases by approximately $10 \%$ when the sample temperature increases from 5.0 to $7.0 \mathrm{~K}$. 


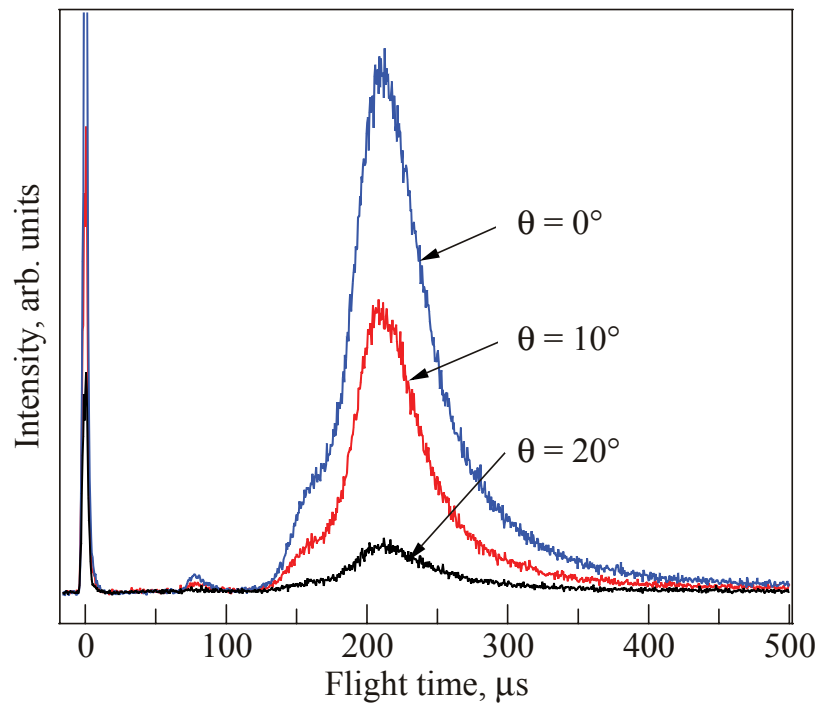

Fig. 3. (Color online) Time-of-flight spectra of metastable atoms desorbed from the surface of annealed solid Ne measured at $\theta=0,10$ and $20^{\circ}$. The incident electron energy was $30 \mathrm{eV}$ and the thickness of the sample was 300 atomic layers.

\section{Annealing effect}

Our results for the time-of-flight spectra and the angular distribution indicate that

- the signal intensity detected in the sample normal direction increases in the annealed sample,

- the angular distribution of the unannealed sample is broader than that of the annealed sample, and

- the kinetic energy of the desorbed metastable atoms increases in the annealed sample.

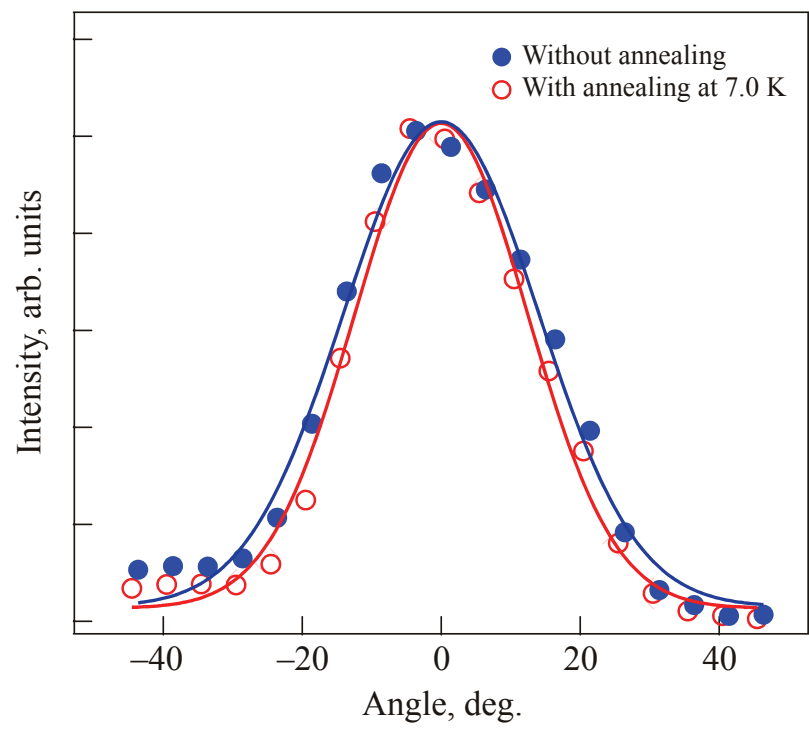

Fig. 4. (Color online) Angular distribution of desorbed metastable $\mathrm{Ne}$ atoms through the $\mathrm{CE}$ mechanism. Solid and open circles are the results measured with an unannealed sample and a sample annealed at $7.0 \mathrm{~K}$, respectively. Solid lines are the best-fitting curves using a Gaussian fitting function.

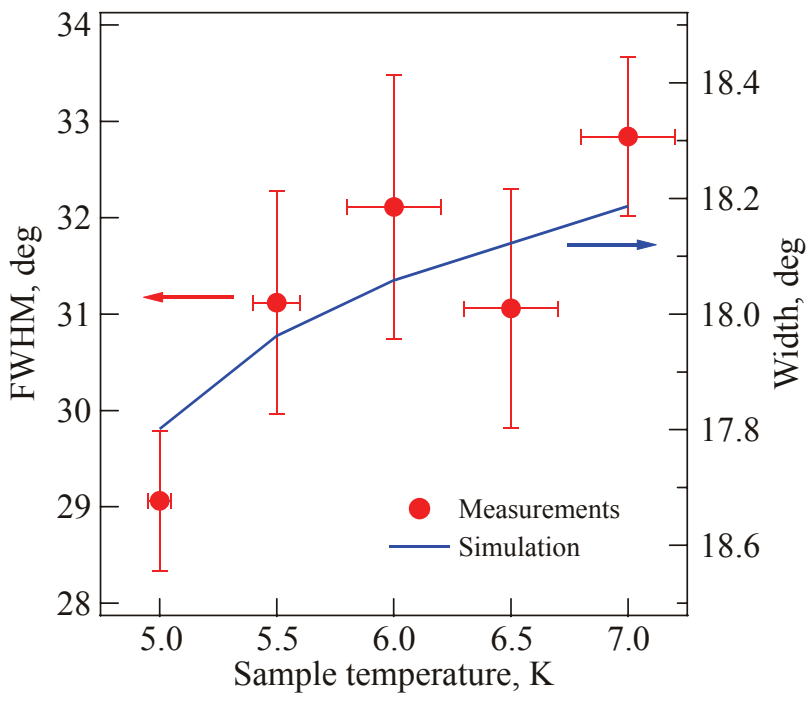

Fig. 5. (Color online) FWHM of the angular distribution of desorbed metastable $\mathrm{Ne}$ atoms through the $\mathrm{CE}$ mechanism as a function of the sample temperature (solid circles). The solid line is the result of our simple trajectory calculation. See text for details.

The motive force associated with desorption through the $\mathrm{CE}$ mechanism is the repulsive force from ground state atoms surrounding the excited atom, which should be axially symmetric around the sample normal direction. The desorbed excited atoms should, therefore, exhibit a narrow angular distribution towards the sample normal direction, as observed in the present study. If the surface is rough, as shown in Fig. 6,a, which is the case if the sample is formed at low temperatures, atoms desorb in the direction normal to the local surface. This results in a broadening of the angular distribution, as seen in Fig. 4. This also explains why the signal intensity detected in the sample normal direction increases when the sample is annealed.

Cui et al. pointed out, based on molecular dynamics calculations [6], that the kinetic energy of excited atoms desorbed from the surface of solid Ar increases as the number of surrounding atoms increases. It can be reasonably assumed that the number of surrounding atoms in the annealed sample - see Fig. 6,b- is larger than that in the unannealed sample: see Fig. 6,a. Therefore we attribute the increase in the kinetic energy in the annealed sample to the flatness of the sample surface, i.e., the average number of atoms surrounding the excited (desorbing) atom.
Unannealed sample

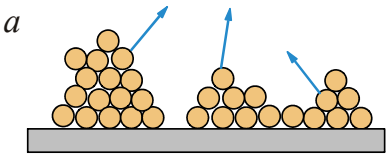

\section{Annealed sample}

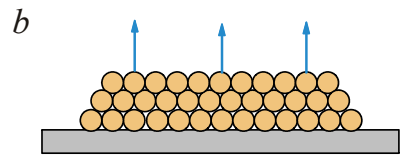

Fig. 6. (Color online) Schematic of the surface of the unannealed (a) and the annealed (b) sample. Solid circles represent $\mathrm{Ne}$ atoms and the arrows point in the direction of the desorption through the CE mechanism. See text for details. 


\section{Temperature effect}

The present results show that the width of the angular distribution becomes broader as the sample temperature increases. Sakurai et al. [2] pointed out, based on their simple trajectory calculation, that the angular spread cannot be explained by the zero-point energy of the atom at the lattice point alone, but it is essentially caused by vibrational displacement of $\mathrm{Ne}$ atoms around the lattice point. Therefore we attribute the broadening of the angular distribution at higher sample temperatures to an increase in the vibrational amplitude. To confirm this idea, we performed a simple trajectory calculation, which will be discussed below.

\section{Trajectory calculation}

The trajectory of desorbing excited atoms that are created at the (111) surface of solid $\mathrm{Ne}$ is calculated as described in [2]. Briefly, an excited $\mathrm{Ne}$ atom in the $2 p^{5} 3 s\left({ }^{1} P_{1}\right)$ state is surrounded by atoms in the ground state $\left({ }^{1} S_{0}\right)$. The potential surface around the excited atom is calculated by superposition of the pair potential between the excited atom and the ground state atoms. The potential between ground state atoms is calculated using the Lennard-Jones $6-12$ potential, and that between $\mathrm{Ne}^{*}\left({ }^{1} P_{1}\right)$ and $\mathrm{Ne}\left({ }^{1} S_{0}\right)$ is calculated based on the theoretical results of Kunsch and Coletti [7]. Ground state atoms within a distance equal to twice the lattice constant (36 atoms) are included in the potential calculation. It is assumed that the ground state atoms do not move during desorption of the excited atom.

The potential thus calculated is inserted into a SIMION potential array file [8]. The trajectory of a desorbing excited $\mathrm{Ne}$ atom is calculated by placing a positive ion with a kinetic energy $E_{0}$ at a distance $R(T)$ from the lattice point. $R(T)$ is estimated as

$$
R(T)=\sqrt{\frac{2\left(E_{0}+\frac{3}{2} k T\right)}{K}}
$$

and $K$ is a constant calculated using

$$
\frac{1}{2} K x_{0}^{2}=E_{0},
$$

where $x_{0}=0.032 \mathrm{~nm}$ and $E_{0}=0.01 \mathrm{eV}$ are the mean square amplitude and the energy of the zero-point vibration in the $\mathrm{Ne}$ crystal, respectively [9]. Ions are distributed along a circle of radius $R(T)$ at intervals of $15^{\circ}$ and the direction of the initial motion is towards and outwards from the center of the circle, i.e., the lattice point.

The calculated angular spread as a function of temperature is shown as the solid line in Fig. 5. Although the calculated angular spread is about $60 \%$ of the measured FWHM, our calculation qualitatively reproduces the experimental temperature dependence.

\section{Summary}

We have measured the time-of-flight spectra and the angular distribution of metastable $\mathrm{Ne}$ atoms desorbed from the surface of annealed and unannealed solid Ne at temperatures between 5.0 and $7.0 \mathrm{~K}$ by low energy electron impact. The dependence on the sample surface conditions and temperature found in the present work are well explained in the framework of the CE mechanism. A simple trajectory calculation qualitatively reproduced the results obtained here.

\section{Acknowledgments}

One of us (T.H.) is grateful to Dr. Elena V. Savchenko for her valuable discussions and comments as well as continuous encouragement since we first met in 1994. This work was partially supported by a Grant-in-Aid for Scientific Research from the Ministry of Education, Culture, Sports, Science and Technology, Japan, and by the Rikkyo University Special Fund for Research (Rikkyo SFR).

1. For recent review, see T. Hirayama and I. Arakawa, J. Phys. Cond. Matter 18, S1563 (2006) and references therein.

2. J. Sakurai, T. Nagai, M. Abo, T. Hirayama, and I. Arakawa, J. Vac. Soc. Jpn. 38, 414 (1995).

3. S. Fujita, K. Fukai, T. Tachibana, T. Koizumi, and T. Hirayama, J. Phys. Conf. Ser. 163, 012083 (2009).

4. K. Fukai, S. Fujita, T. Tachibana, T. Koizumi, and T. Hirayama, J. Phys. Cond. Matter 22, 084007 (2010).

5. T. Tachibana, K. Fukai, T. Koizumi, and T. Hirayama, J. Phys. Cond. Matter 22, 475002 (2010).

6. S. Cui, R.E. Johnson, and P. Cummings, Phys. Rev. B39, 9580 (1989).

7. P.L. Kunsch and F. Coletti, J. Chem. Phys. 70, 726 (1979).

8. http://simion.com/

9. H.R. Lyde, in: Rare Gas Solid, M.L. Klein, and J.A. Venables (eds.), Academic Press, London (1976), Vol. 1, p. 382. 